\title{
Enhanced Motor Learning in Older Adults Is Accompanied by Increased Bilateral Frontal and Fronto-Parietal Connectivity
}

\author{
Chien-Ho Janice Lin, ${ }^{1,2,{ }^{*}}$ Ming-Chang Chiang, ${ }^{3,{ }^{*}}$ Allan D. Wu, ${ }^{1,2}$ Marco lacoboni, ${ }^{2,4}$ Parima Udompholkul, ${ }^{1}$ \\ Omid Yazdanshenas, ${ }^{5}$ and Barbara J. Knowlton ${ }^{5}$
}

\begin{abstract}
We recently demonstrated that older adults can benefit as much as younger adults from learning skills in an interleaved manner. Here we investigate whether optimized learning through interleaved practice (IP) is associated with changes in inter-regional brain connectivity and whether younger and older adults differ in such brainbehavior correlations. Younger and older adults practiced a set of three 4-element motor sequences in a repetitive or in an interleaved order for 2 consecutive days. Retention of the practiced sequences was evaluated 3 days after practice with functional images acquired simultaneously. A within-subject design was used so that subjects practiced sequences in the other condition (repetitive or interleaved) 2-4 weeks later. Using the psychophysiological interaction (PPI) analysis approach, we found that IP led to higher functional connectivity between the right and left dorsal lateral prefrontal cortex (DLPFC) and between the dorsal premotor cortex (PMd) and inferior parietal lobule (IPL) in older adults. Moreover, increased connectivity between these regions was significantly associated with the learning benefits of IP. In contrast, in younger adults, enhanced learning as a result of IP was associated with increased connectivity between DLPFC and the supplementary motor area (SMA) and the inferior frontal gyrus. These data suggest that though younger and older gain similar behavioral benefits from interleaved training, aging may alter the operation of brain networks underlying such optimized learning.
\end{abstract}

Key words: aging; contextual interference; functional connectivity; fMRI; practice schedule; psychophysiological interaction; serial reaction time task

\section{Introduction}

D ESPITE THE FACT that cognitive functions decline with age, the healthy elderly can exhibit robust learning (e.g., Lin et al., 2010), through employment of different neural circuits from younger adults to accomplish the same task goals (Wolk et al., 2009). For example, older adults recruited frontal regions bilaterally during text recall, while frontal activity in younger adults was more unilateral (Cabeza et al., 1997). In working memory tasks, older adults who retrieved their memory faster showed greater activity in the dorsal lateral prefrontal cortex (DLPFC) than those who did slower, while the same comparisons in younger adults showed the opposite pattern (Rypma and D'Esposito, 2000). These studies support the premise that aging alters the activation pat- terns of local brain regions, and that these regions interact to function globally as a network (Klostermann et al., 2012).

Previous investigations show that practicing tasks with sufficient difficulty leads to better learning, a strategy called desirable difficulty. An example of desirable difficulty is the contextual interference $(\mathrm{CI})$ paradigm, where the difficulty level of the practice context is increased by presenting multiple tasks in an interleaved (random) order compared to a repetitive (blocked) order (Shea and Morgan, 1979). In our previous study, we found that practicing tasks in an interleaved order (interleaved practice [IP]) led to inferior performance during practice, but superior learning performance as measured on a retention test compared to repetitive practice (RP). Moreover, this learning benefit was associated with both increased functional activity in the DLPFC and the dorsal premotor cortex

\footnotetext{
${ }^{1}$ Department of Neurology, David Geffen School of Medicine, University of California, Los Angeles, Los Angeles, California.

${ }^{2}$ Ahmanson-Lovelace Brain Mapping Center, University of California, Los Angeles, Los Angeles, California.

${ }^{3}$ Department of Biomedical Engineering, National Yang-Ming University, Taipei, Taiwan, R.O.C.

${ }^{4}$ Department of Psychiatry and Biobehavioral Sciences, University of California, Los Angeles, Los Angeles, California.

${ }^{5}$ Department of Psychology, University of California, Los Angeles, Los Angeles, California.

*These two authors contributed equally to this work.
} 
(PMd), and increased electrophysiological excitability of the primary motor cortex (M1) (Lin et al., 2011). These findings suggest that motor learning involves functional integration between motor-related areas, such as DLPFC, PMd, and M1.

In this article, we went a step further to explore functional connectivity networks with respect to DLPFC and PMd, and their association with the CI benefit to learning. We were also interested in how the connectivity patterns of these networks change with age. Functional brain connectivity refers to temporal correlations and causal interactions between the neuronal activities of two distinct brain regions (Rubinov and Sporns, 2010) and may be quantitatively assessed using the psychophysiological interaction (PPI) method (Friston et al., 1997). PPI has been extensively used in neuroimaging research, and is commonly accepted as a powerful way to characterize discrete functional networks for the encoding, recall, and recognition phases of memory tasks (Rissman et al., 2004). In our study, healthy younger and older adults practiced motor sequences over two consecutive days, and then were tested with trained sequences 3 days after practice, while functional imaging data were acquired simultaneously. We examined functional connectivity with respect to DLPFC and PMd in older and younger adults during the retrieval of motor sequences learned under interleaved compared with those learned under RP. Given that the strength of brain functional connectivity is a function of learning (Ma et al., 2010), we hypothesized that practice under CI conditions may increase prefrontal and premotor connectivity with other brain regions, which in turn, enhances retrieval of sequences. Through the PPI analysis, we found that older and younger adults relied on different patterns of functional connectivity for this benefit. To our knowledge, this is the first study investigating how aging may modulate the cortical networks underlying learning that has been optimized by contextual interference.

\section{Materials and Methods}

\section{Participants}

Sixteen older (seven men and nine women, age $=66.2 \pm 4.7$ years, education $=16.5$ years; mean \pm standard deviation) and sixteen younger (nine men and seven women, age $=26.4 \pm 3.1$ years, education $=17.2$ years) right-handed adults participated the study. They were recruited from the University of California, Los Angeles, and the adjacent community. Participants were excluded if they were a musician or a professional typist, scored $<28$ on the Mini-Mental State Exam (Folstein et al., 1975), had any contraindications to magnetic resonance imaging (MRI), uncorrected vision loss, neuromuscular dysfunction, or significant medical, neurological, or psychiatric condition that prevented them from performing the task. All participants gave informed consent using an institutionally approved consent form. The gender distribution and the level of education were not significantly different between the two groups ( $p=0.57$ and $p=0.42$, respectively).

\section{Behavioral task}

The behavioral experiment was arranged in a withinsubject cross-over design, where the participants practiced the serial reaction time (SRT) task (Cross et al., 2007; Wright et al., 2005) over a course of 5 days. Each trial of the SRT task required the participants to make a keypress response as quickly and accurately as possible to successive elements of a spatial sequence. The participants positioned the four fingers of their left hand (except the thumb) on the four lightsensitive response keys of a magnet-compatible button box (Current Designs, Inc.). They were asked to press the key corresponding to the location of a colored circle appearing through magnet-compatible goggles (Fig. 1A); the next colored circle showed up immediately after the keypress was made. One colored circle appeared at a time, while the circles in the other positions remained transparent. The same color (yellow, red, blue, or green) was always displayed at the same circle location, providing a consistent spatial and color cue. To make certain that each participant practiced an equal number of trials, each trial was set to a fixed duration of $3 \mathrm{sec}$. If the participants finished the four keypresses in response to a test sequence before $3 \mathrm{sec}$, four transparent circles would appear on the screen to maintain visual stimulation before the next trial (test sequence) (Fig. 1A). An in-house computer program running under Presentation software (Neurobehavioral Systems) controlled the order of appearance for the colored circles and recorded the participants' response time (RT), defined as the sum of every interval between visual stimulus onset and keypressing. The nondominant hand (left hand) was used to increase the difficulty level of the task and enhance the opportunity for learning. This strategy has been applied previously in studies using short-sequence SRT finger-tapping tasks (e.g., Cross et al., 2007; Lin et al., 2011). For days 1 and 2, there were three practice sessions on each day. Participants practiced the SRT task in either the RP or the IP condition. Participant started in either the RP or the IP condition in the first week; 2 weeks later, the participants crossed over to the other condition (i.e., $\mathrm{RP} \rightarrow \mathrm{IP}$, or vice versa). The order of the practice conditions and the SRT sequences was counterbalanced across the participants. For the RP, each of the three 4-item test sequences was practiced repetitively for 54 consecutive trials before the next sequence appeared. For the IC, the three test sequences were arranged in an interleaved or nonrepetitive manner within the 162 trials (Fig. 1A), and the same arrangement of the test sequences was applied to all the participants to ensure that every participant in the IP condition performed the same order of test sequences. Three days after practice (day 5), retention, that is, learning of the SRT task, was evaluated based on reduction in the RT (Fig. 1C). Retention tests took place in an MRI scanner with two retention sessions; each corresponds to a functional imaging run (Fig. 1C).

\section{Functional magnetic resonance imaging}

During the retention tests, the participants performed the SRT task within a Siemens Trio 3.0 T MRI scanner at the Ahmanson-Lovelace Brain Mapping Center at UCLA, while functional magnetic resonance imaging (fMRI) data were acquired concurrently. The fMRI experiment was arranged in a blocked design, with each 18-second task block followed by an 18 -sec rest block. Since the test sequence in each SRT trial (one sequence per trial) was displayed for a fixed duration of $3 \mathrm{sec}$, each fMRI task block contained six trials. During the rest block, the circles were replaced by a fixation cross in the center of the screen, and the participants were instructed to gaze at the fixation cross while staying relaxed. 


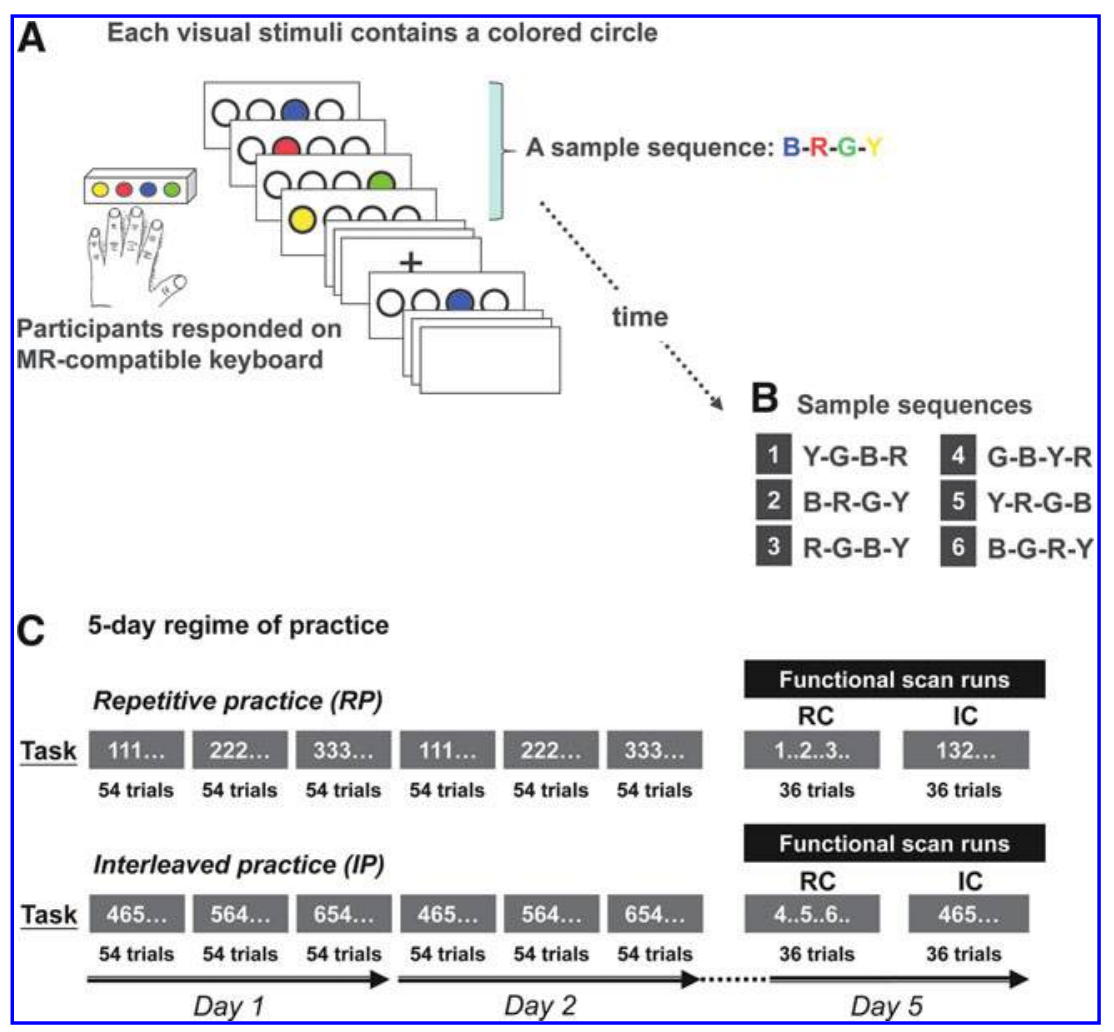

FIG. 1. RP and IP in the SRT sequence-learning task. The 5-day regime of the SRT sequence-learning task is shown in (C). The participants practiced tasks on days 1 and 2. In each trial of the SRT task, they had to respond to one of the three different fourelement sequences, denoted by 1, 2, or 3 (B), with each element represented by a circle colored in yellow (Y), red (R), blue (B), or green $(\mathrm{G})$ appearing through the magnet-compatible goggles (A). The participants were asked to press the corresponding key using their left hand as soon as they saw one of the colored circles. To investigate the effects of contextual interference on learning, we assigned each participant to either the RP or the IP condition. In the RP condition, each of the three test sequences was practiced repetitively for 54 consecutive trials before the next sequence appeared [e.g., $111 \ldots .222 \ldots 333 \ldots$; see (C) top]. For the IP condition, the three test sequences were arranged in an interleaved or nonrepetitive manner [(C), bottom]. On day 5, the participants were tested for their learning performance with the trained sequences while functional imaging data were acquired concurrently. For both RP and IP participants, the practiced sequences were presented in a repetitive order in the first fMRI run (denoted by RC; in the second run they were presented in an interleaved order (denoted by IC). Two weeks after practice and retention for one training condition, the participants returned to practice a different set of three sequences in the other condition (e.g., $\mathrm{RP} \rightarrow \mathrm{IP}$, or vice versa). RP, repetitive practice; IP, interleaved practice; SRT, serial reaction time; RC, repetitive test condition; IC, interleaved test condition; fMRI, functional magnetic resonance imaging.

The participants underwent two fMRI runs during the retention tests, with six task-rest block pairs lasting for $216 \mathrm{sec}$ per run (Fig. 1A, day 5), where they were tested with the same three sequences as they had practiced on days 1 and 2, i.e., each sequence appeared 24 times over the two runs. In the first run, the practiced sequences were presented in a repetitive order (denoted by repetitive test condition, or RC), and in the second run, they were presented in an interleaved order (denoted by interleaved test condition, or IC). This was to ensure that practice-test compatibility was equal for either practice condition (Lee, 1988; Lee and Magill, 1983; Shea and Morgan, 1979). The order of these runs was counterbalanced across participants. Each sequence was presented for 24 trials across these two runs on day 5 . The number of trials for each sequence was reduced (compared to 54 trials during practice days) to limit further learning of the sequences (Cross et al., 2007).

Each fMRI run consisted of 99 echo planar imaging (EPI) volumes (gradient-echo, $\mathrm{TR}=2000, \mathrm{TE}=30$, flip angle $=90^{\circ}$ ), each with 34 transverse slices, 4-mm thickness/1-mm gap, and a $64 \times 64$ matrix, yielding an in-plane resolution of $3 \mathrm{~mm} \times$ $3 \mathrm{~mm}$. The first four volumes of each fMRI run were removed from analysis to allow for magnetization to reach equilibrium. Two sets of high-resolution anatomical images were acquired for image registration: 1. an magnetization-prepared rapid acquisition with gradient echo (MP-RAGE) structural volume $\left(\mathrm{TR}=1,900, \mathrm{TE}=2.26\right.$, flip angle $\left.=8^{\circ}\right)$ with 176 sagittal slices, $1-\mathrm{mm}$ thickness/0.5-mm gap, a $256 \times 256$ matrix, and $1.33 \mathrm{~mm} \times 1.33 \mathrm{~mm}$ in-plane resolution, and 2. a T2-weighted co-planar volume $\left(\mathrm{TR}=5000, \mathrm{TE}=34\right.$, flip angle $\left.=90^{\circ}\right)$ with 34 transverse slices covering the whole brain, 4-mm thickness/ 1-mm gap, a $128 \times 128$ matrix, and an in-plane resolution of $1.5 \mathrm{~mm} \times 1.5 \mathrm{~mm}$.

\section{Data processing and statistical analysis}

In our previous brain mapping study on young adults (Lin et al., 2011), we found CI benefits in motor sequence learning, 
where IP led to better retention than the RP. In this article, we aimed to further determine whether CI may also improve learning in older adults. Therefore, we compared differences in learning between age groups by analyzing the behavioral and fMRI data acquired during the retention test only.

Behavioral data. Behavioral performance was assessed based on the RT for keypressing (see definition in Behavioral Task). The median of RT across every six consecutive trials was used for subsequent analyses.

We examined Age $\times$ Practice condition interactions in the mean RT of the practiced sequences during the retention test on day 5 using a repeated measures analysis of variance (ANOVA) model with Age as the between-subject factor and Practice condition as the within-subject factor. For all statistical tests involving the behavioral data, significance level was set to $p<0.05$. SPSS 13.0 (SPSS Inc.) was used for the statistical analyses.

Functional image processing and analysis. Functional images were processed using Statistical Parametric Mapping software (SPM8, http://fil.ion.ucl.ac.uk/spm/software/spm8/). To correct for motion artifacts, functional image data of each subject were realigned to the first volume in each fMRI run with fourth-degree B-Spline interpolation resampling (Friston et al., 1995). None of the subjects had scans with head motion $>2 \mathrm{~mm}$. The mean image of the realigned volumes for each run was normalized to the standard Montreal Neurological Institute (MNI) EPI template (Evans et al., 1993), with the resulting normalization parameters applied to all the re-aligned volumes of the same run. The normalized images were then resampled to $3 \times 3 \times 3 \mathrm{~mm}^{3}$ per voxel, and subsequently spatially smoothed with an isotropic Gaussian filter with full width at half maximum $=8 \mathrm{~mm}$ before entered into the general linear model (Friston et al., 1995). The fMRI data were modeled using a boxcar function convolved with the hemodynamic response function (HRF). The mean RT of each task block was added as an additional regressor to ensure that any differences in brain activity during practice and retention were due to the influences of the practice conditions, but not to differences in RT.

We next sought to determine whether the differences in the DLPFC or PMd connectivity between IP and RP derived from the primary PPI analysis were influenced by age. For each participant, the primary PPI analysis yielded a contrast map that identified regions where the interleaved condition significantly increased the connectivity of these regions with DLPFC or PMd. We then further tested whether such interaction effects of the interleaved condition differed between the younger and older adults. In other words, we were testing whether the younger adults had a greater interleavedminus-repetitive difference in functional connectivity than the older adults, or vice versa. To do this, we compared the above PPI contrast maps between the younger and older groups, using a second-level random-effects model, where the group difference in PPI contrasts was estimated as a fixed variable, while the population variance of the PPI contrasts was modeled as a random effect (Friston et al., 2005). We focused on the right $\operatorname{DLPFC}(24,3,51)$ and the right PMd $(24,-2,56)$ here, as we found higher blood-oxygen level-dependent (BOLD) activity in these regions during IP compared to RP in younger adults (Lin et al., 2011). Moreover, performing sequences in an interleaved condition in- volves more motor planning and executive ability, the cognitive processes known to be mediated by the DLPFC and the PMd (Brady, 2008; Lin, et al., 2011).

Psychophysiological interaction analysis. We used the PPI method (Friston et al., 1997; Gitelman et al., 2003) to assess the hypothesis that IP led to different patterns of prefrontal and premotor connectivity during retention between younger and older adults. The PPI method was designed to model how neural activity in one brain region, i.e., the seed region, differently interacts with that in another brain region, or the target region, when there are changes in a cognitive/perceptual process. We selected the PPI analysis, as it measures the influence one neural system exerts over another (Friston, 1994), under the modulatory effects of cognitive task conditions; e.g., RP or IP conditions in our article. Other brain connectivity techniques are available [see ( $\mathrm{Li}$ et al., 2009) for a review of these methods], and the simplest one may be to compute the cross-correlation of the BOLD time series between two brain regions. Nevertheless, we were interested in how different learning conditions may change the connectivity between key regions in the brain motor network, and as such the PPI analysis appeared to be the best fit to estimate task-dependent brain functional connectivity as demonstrated in our study.

Specifically, our PPI model included three regressors in the design matrix: 1. the psychological variable, representing the cognitive/perceptual process of interest, or the IP versus the RP here, 2 . the physiological variable, representing the BOLD signal time series in the seed regions in the right DLPFC or the right PMd, and 3. the interaction term of 1 and 2 . The dependent variable is the BOLD signal time series in the target region.

fMRI time-series data within a sphere of radius $6 \mathrm{~mm}$ around the peak activation voxel of the seed region in the right DLPFC or the right PMd were extracted from each participant's normalized data. The time-series data were deconvolved with the HRF and then mean-corrected and high-pass filtered (cutoff $=128 \mathrm{sec}$ ) to remove low-frequency drifts (Mohanty et al., 2008). The product of the activation time-series data at the seed region and the psychological vector of interest resulted in the PPI interaction term. To explore functional connectivity with respect to the right DLPFC or the right PMd without a priori assumption for possible target regions, we performed a voxel-wise PPI analysis, where every voxel outside the seed region was set as a target region for the PPI regression. This led to a statistical parametric map for each participant, showing regions where the PPI interaction term was significant; i.e., the regression slopes for the neural activity (deconvolved fMRI data) between the seed and the target regions were significantly different across different practice conditions. All the individuallevel PPI statistical parametric maps were then entered into a one-sample $t$-test under a random-effects model, to account for intersubject variability in functional connectivity patterns. Multiple comparisons across the brain were corrected using the topological false-discovery rate (FDR) method (Chumbley et al., 2010). The FDR of significant clusters (defined as sets of contiguous voxels with significant effects) were controlled to be within $5 \%$, indicating that on average $<5 \%$ of clusters that were considered significant tended to be false positive. We further set an additional 
threshold on the cluster size, where only clusters of more than six voxels were displayed.

Age influences on condition-dependent functional connectivity. We next sought to determine whether the differences in the DLPFC or PMd connectivity between IP and RP derived from the primary PPI analysis were influenced by age. Using a random-effects model, we compared the PPI effects between the younger and older groups in regions where increased connectivity with the DLPFC or PMd in the interleaved condition was identified in the previous section. Multiple comparisons were corrected based on the topological FDR and cluster-size criteria as described above.

Associations between learning and condition-dependent functional connectivity. To explore whether differences in connectivity between the IP and RP conditions were functionally significant, we correlated the differences in the DLPFC or PMd connectivity between IP and RP with learning performance on the retention testing. Here, we tested a directional hypothesis that increase in the DLPFC or the PMd connectivity correlated with learning benefit following IP, as we found that IP led to greater functional connectivity (see Results) and better learning performance [see (Lin et al., 2011)] on retention. Learning benefit was defined as the reduction in the mean RT during retention of the practiced sequences on day 5, comparing the IP to the RP condition; a positive R-I (repetitive minus interleaved) contrast in RT indicates a faster motor response, or behavioral improvement, following IP. On the other hand, a positive $\mathrm{I}-\mathrm{R}$ contrast in the functional connectivity between the PMd or DLPFC and other brain regions indicates greater connectivity, or higher regression slope in BOLD time series data, between these regions following the IP.

We used a region-of-interest (ROI)-analysis approach to assess the functional significance of the enhanced brain connectivity following IP. The ROI approach served as a secondary exploration for our primary voxelwise PPI analysis above. In addition to the seed regions within the right DLPFC or PMd used in the primary PPI analyses, we also included regions known to be critical to the functional networks with respect to the DLPFC or the PMd, including inferior parietal cortex for the PMd-seeded networks (Keisker et al., 2009), and supplementary motor area (SMA), inferior frontal gyrus, rostral frontal area, and the contralateral (left) DLPFC for the DLPFC-seeded networks (Marek et al., 2010). Correlations between the I- R contrast in functional connectivity and the $\mathrm{R}-\mathrm{I}$ difference in the mean RT were then tested using the Pearson's correlation method.

\section{Results}

\section{Behavioral results}

At the start of practice on day 1, the mean RT showed no significant difference between the interleaved and repetitive conditions within each age group $[p=0.21, F(1,15)=2.05$, younger; $p=0.25, F(1,15)=1.74$, older, Fig. 2A]. Performance of younger and older participants improved after 2 days of practice, and the mean RT across the two practice days was faster in the repetitive than in the interleaved condition (mean RT in millisecond, younger-repetitive [YR] $=880.8 \pm$ 69.4, younger-interleaved [YI] $=1121.6 \pm 64.7, p=0.006$; older- repetitive $[\mathrm{OR}]=1424.6 \pm 69.4$, older-interleaved $[\mathrm{OI}]=1700.9 \pm$ 64.5, $p=0.038$; Fig. 2A, day 1 and day 2, RP was lower than IP in both younger and older groups). This pattern was, however, reversed on the retention test on day 5 , where performance of the trained sequences was better for the sequences practiced in the interleaved than in the repetitive condition, especially when the trained sequences were presented in an interleaved order [mean RT, YR=1114.9 $\pm 74.4, \mathrm{YI}=897.4 \pm 37.7, p<0.001$, $F(1,15)=8.78 ; \mathrm{OR}=1738.6 \pm 74.2, \mathrm{OI}=1542.6 \pm 68.8, p=0.013$, $F(1,15)=6.88$, Fig. 2B]. All subjects performed above $95 \%$ accuracy at the end of practice and during the retention sessions. These results are consistent with findings of previous work on CI effects based on various paradigms (Brady, 2008), and further demonstrate that motor learning in older adults can also benefit from CI.

\section{fMRI results}

Seed I: right PMd. Figure $3 \mathrm{~A}$ and $\mathrm{B}$ show brain regions where PMd-seeded connectivity was significantly different between the repetitive and interleaved conditions, separately for the younger (Fig. 3A) and older (Fig. 3B) groups during the retention phase on day 5. Significant PPI effects in these regions may be better illustrated by comparing the regression lines of BOLD activities in these regions (y-axis) on the PMd activities ( $x$-axis) between the practice conditions (Supplementary Fig. S1; Supplementary Data are available online at www.liebertonline.com/brain). These regions are also listed in Table 1. For the younger adults, no brain regions showed significant differences between practice conditions in functional connectivity with respect to the PMd during sequence retrieval (Fig. 3A). By contrast, the older adults showed increased connectivity with PMd after the IP in the inferior parietal lobule (IPL), a region classically associated with movement execution and sensorimotor transformation (Karni et al., 1998) (Fig. 3B). This is further demonstrated by the regression plots in Supplementary Figure S1, where the regression slopes of the BOLD signal between PMd and IPL were significantly different between practice conditions in older, but not in younger adults [older, Supplementary Fig. S1, I(B); younger, Supplementary Fig. S1, I(A)]. The nonsignificant effects of IP on PMd connectivity in younger participants (Fig. 3A) suggest that CI does not have long-term effects on PMd connectivity in younger adults. These findings suggest that the PMd contributes to coordinating circuits involved in motor skill retrieval more in older than in younger adults.

Seed II: right PMd. Figure $3 \mathrm{C}$ and D show maps of brain regions (listed in Table 1) where DLPFC-seeded modulations were significantly different between the two practice conditions during the retention phase in both the younger (Fig. 3C) and older (Fig. 3D) adults. Greater DLPFC connectivity during sequence retrieval for the interleaved over the repetitive conditions was present in the right rostral frontal region and the left inferior frontal lobule (IFL) [Fig. 3C, D and Supplementary Fig. S1, II(A), II(E), II(D), and II(H)] regions functionally associated with memory retrieval and response inhibition (Aron et al., 2004; Schwarb and Schumacher, 2009). Comparing PPI effects between the age groups, we found that connectivity of the rostral frontal and the SMA with respect to the DLPFC increased more in the younger than the older adults following IP [Fig. 3C, Supplementary Fig. S1, II(C)]. By 


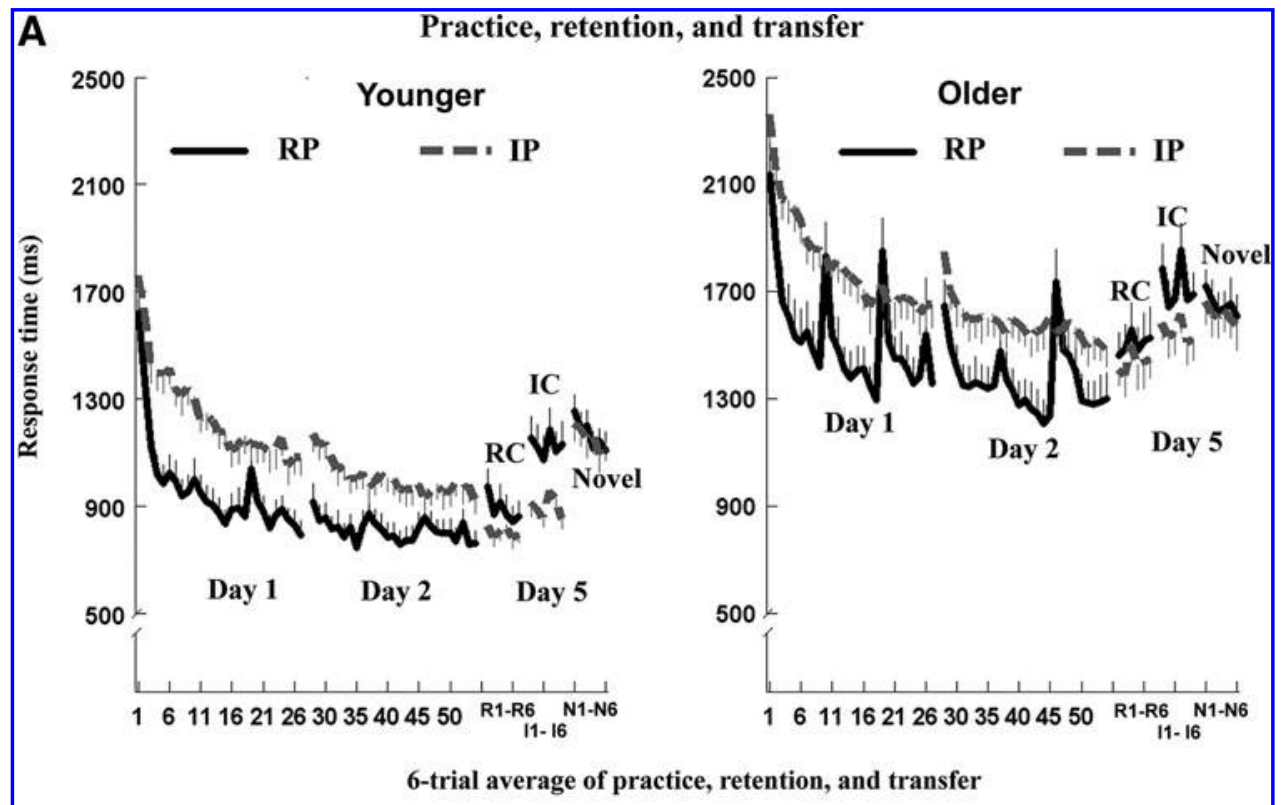

B

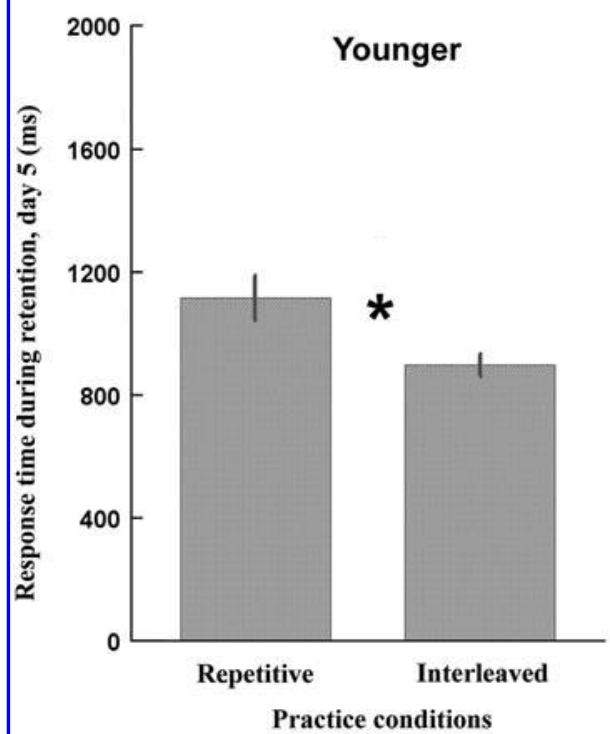

Performance during retention in the interleaved testing condition

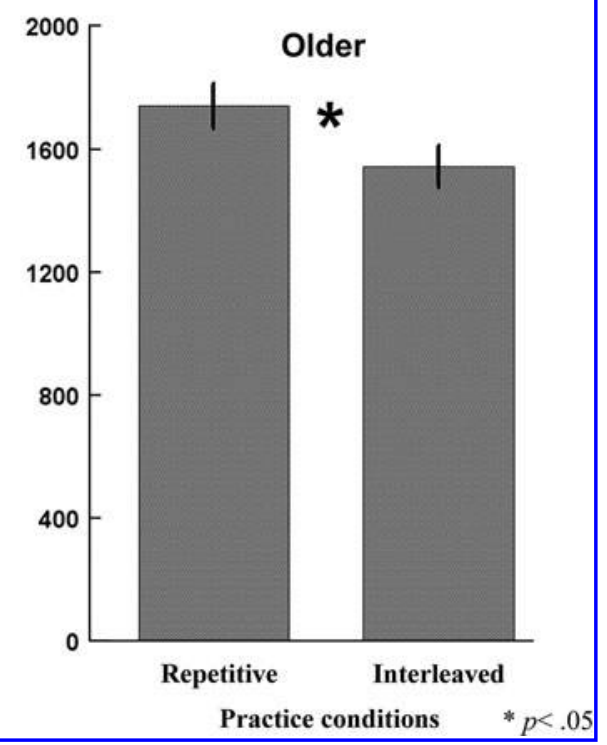

FIG. 2. Comparisons between younger and older adults on SRT task performance under different practice conditions. (A) Illustrates task performance, measured by the RT (mean \pm standard error) in practice on days 1 and 2 (blocks 1-54), and in retention of the trained and novel sequences on day 5 . The trained sequences in the retention tests were further arranged in either an RC or an IC. Sessions for novel sequences are labeled as Novel. R1-R6 on the $x$-axis denote the six RT blocks for the RC, I1-I6 for the IC, and N1-N6 for the Novel sequences. Both younger and older adults improved their performance after 2 days of practice, as shown by the decrease in RT. On comparisons between practice conditions, there was no significant difference in the RT between the RP (solid line) and the IP (gray dotted line) conditions at the beginning of practice. During practice, the RT was shorter in the RC than the IC condition, but during retention on day 5 , the performance of the trained sequences was significantly better for the IP than the RP condition, especially when the testing sequences were presented in an interleaved order (IC) (B). These findings replicate the $\mathrm{CI}$ benefit on motor sequence learning, and here, we have further shown that the CI benefit is present in the elderly population. RT, response time; $\mathrm{CI}$, contextual interference. contrast, greater connectivity between the right and the left DLPFC following IP was detected in the older, but not in younger, adults [Fig. 3D, Supplementary Fig. S1, II(F)]. Overall, these findings suggest that the intrinsic connectivity of motor and higher order cognitive networks was enhanced following interleaved sequence practice in the young and aged brain. This practice effects on DLPFC connectivity were present even 3 days after practice in both groups, suggesting that retrieval of the practiced sequences is associated with the functional integration of the DLPFC and brain regions known for memory retrieval and executive control (Fig. 3C, D). In addition, interhemispheric DLPFC connectivity, and enhanced sensorimotor integration resulting from IP may play an important role in skill retrieval later in the lifespan.

Age $\times$ Practice condition interaction in functional connectivity. We then performed a two-way ANOVA to deter- mine whether an Age $\times$ Practice condition interaction was present in functional connectivity during sequence retrieval. IP led to stronger functional connectivity in the older than the younger groups, between the right PMd and the IPL [Fig. 4A(1)], the right DLPFC and the left DLPFC (Fig. 4B1), and the right DLPFC and the left inferior frontal gyrus [Fig. $4 \mathrm{~B}(2)]$. By contrast, IP increased the connectivity between the right DLPFC and SMA more in the younger adults than the older adults [Fig. 4B(3)]. These findings show that brain networks underlying the CI benefits in motor sequence learning differ with age, and that aging influences the recruitment of neural substrates for memory consolidation and retrieval.

Increased PMd and DLPFC connectivity may indicate efficient retrieval. We next conducted an exploratory analysis to observe whether increased connectivity following IP was associated with $\mathrm{CI}$ benefits on retention, defined as a shorter 


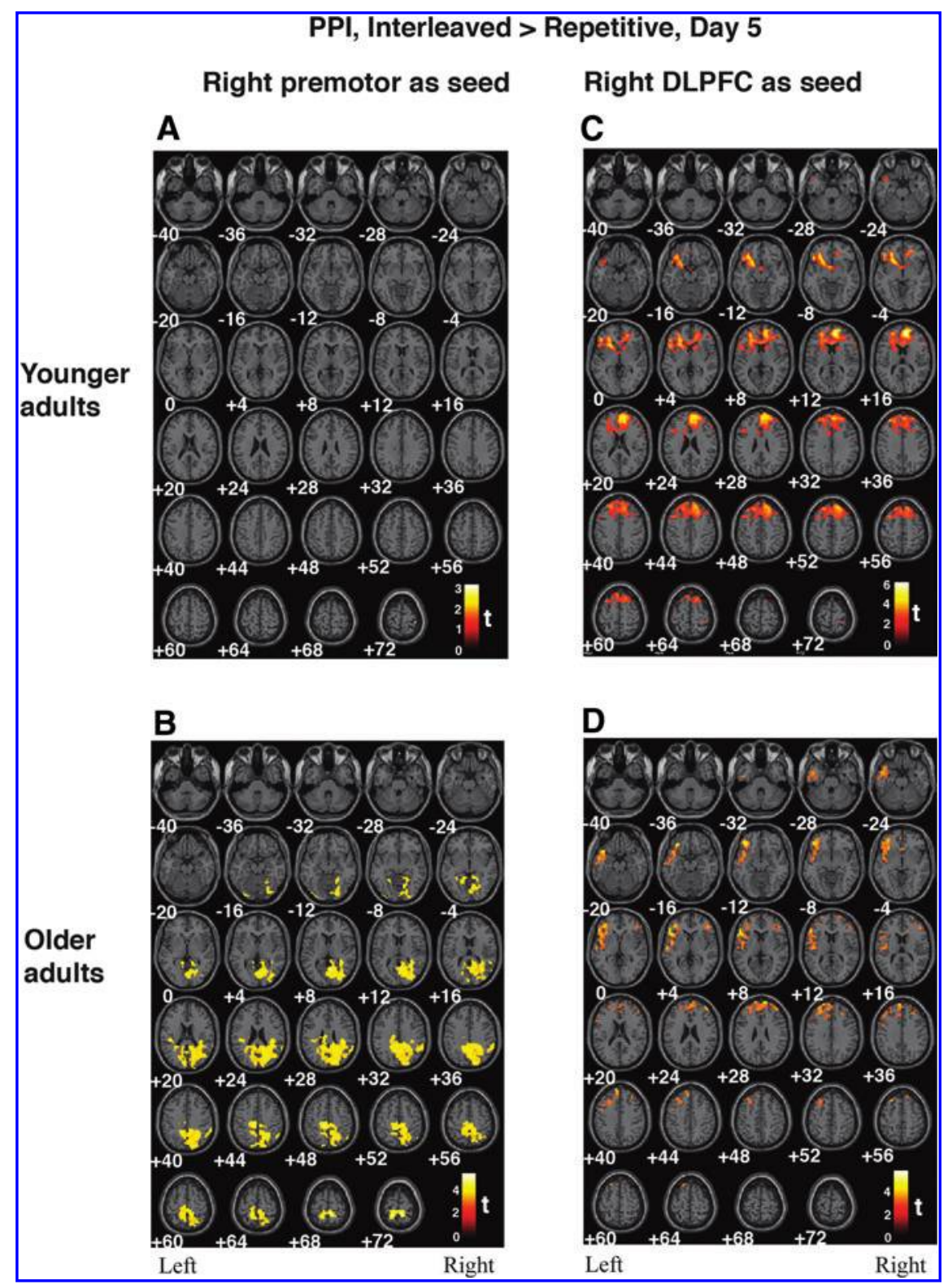

FIG. 3. Effects of practice conditions on brain functional connectivity during the retention phase for younger and older adults. To identify the differences in functional connectivity during retention between the RP and the IP conditions, the fMRI BOLD time series at each voxel was regressed against that of the right PMd $(\mathbf{A}, \mathbf{B})$ or the right DLPFC (C, D), with the IC or RC as the interaction term, and the between-participant variability as the random-effects variable. The resulting significance maps are displayed in neurological orientation, with the MNI coordinate (in $\mathrm{mm}$ ) at the bottom. Colored regions, where voxel $t$-values are positive, indicate that IP had significantly greater interaction effects than RP on the functional connectivity between that region and the right PMd (left column) or the right DLPFC (right column) cortex, corrected for multiple comparisons using the topological false-discovery rate method. Both age groups showed higher DLPFC functional connectivity during sequence retrieval on day 5 following the IP $(C, D)$, but in different brain areas. IP led to stronger connectivity with respect to the right DLPFC in the anterior and inferior frontal regions for the younger adults $(\mathrm{C})$, but in the anterior frontal region, the left IFL and the left DLPFC for the older adults (D). On the PMd connectivity, although we did not detect any interaction effects of practice condition in the younger adults (A), we found that the older adults showed higher connectivity following the IP between the PMd and regions involving sensorimotor planning; e.g., the postcentral gyrus and the inferior parietal areas (B). These results show that stronger functional couplings in regions of higher order executive function developed in both age groups as a result of IP (C, D). Moreover, aging may interact with practice conditions to modulate functional brain networks that are essential for successful sequence retrieval-the older adults may uniquely need to exploit cortical motor networks during sequence retrieval (B) as an alternative sensorimotor strategy to better retrieve the practiced tasks. PMd, dorsal premotor cortex; DLPFC, right dorsal lateral prefrontal cortex; MNI, Montreal Neurological Institute; IFL, inferior frontal lobule. 
Table 1. Brain Areas Showing Positive Change in Coupling with the Right Premotor (Upper Section) and Right Dorsal lateral Prefrontal Cortex (Lower Section) During Day 5 Retention After Sequences Were Practiced in Interleaved Versus Repetitive Conditions

\begin{tabular}{|c|c|c|c|c|c|}
\hline Regions & $x$ & $Y$ & $z$ & $T$ & $p$ \\
\hline \multicolumn{6}{|c|}{ Older adults, coupling with Rt PMd } \\
\hline Lt paracentral lobule & -12 & -39 & 75 & 5.34 & $<0.001$ \\
\hline Rt paracentral lobule & 9 & -42 & 75 & 5.33 & $<0.001$ \\
\hline Rt precuneus & 9 & -50 & 9 & 5 & $<0.001$ \\
\hline Lt inferior temporal gyrus & -54 & -69 & -6 & 4.78 & $<0.001$ \\
\hline Lt inferior occipital gyrus & -42 & -87 & -9 & 4.16 & $<0.001$ \\
\hline Lt thamalus & -3 & -24 & 6 & 3.82 & 0.001 \\
\hline Rt cerebellum & 9 & -57 & -51 & 3.71 & 0.001 \\
\hline Lt cerebellum & -3 & -57 & -51 & 3.43 & 0.002 \\
\hline Lt medial temporal gyrus & -48 & -27 & -6 & 3.61 & 0.001 \\
\hline Lt caudate & -35 & -27 & -9 & 3.32 & 0.002 \\
\hline Lt insular & -39 & -21 & 3 & 3.32 & 0.002 \\
\hline Rt precentral gyrus & 57 & -12 & 45 & 3.3 & 0.002 \\
\hline Lt parahippocampal gyrus & -30 & -18 & -18 & 3.28 & 0.003 \\
\hline Rt postcentral gyrus & 60 & -21 & 42 & 3.23 & 0.003 \\
\hline Lt superior frontal & -21 & -6 & 75 & 3.22 & 0.003 \\
\hline Rt thalamus & 18 & -18 & 15 & 3.21 & 0.003 \\
\hline Lt angular gyrus & -48 & -78 & 27 & 3.2 & 0.003 \\
\hline Lt fusiform gyrus & -54 & -3 & -30 & 3.11 & 0.004 \\
\hline Lt inferior parietal lobule & -57 & -54 & 42 & 3.04 & 0.004 \\
\hline Lt superior parietal lobule & -36 & -66 & 57 & 3.01 & 0.004 \\
\hline \multicolumn{6}{|c|}{ Older adults, coupling with Rt DLPFC } \\
\hline Lt inferior frontal gyrus & -36 & 33 & -15 & 5.9 & $<0.001$ \\
\hline Lt superior temporal gyrus & -42 & 15 & -21 & 3.54 & $<0.001$ \\
\hline Lt medial frontal gyrus & -6 & 57 & 39 & 3.55 & $<0.001$ \\
\hline Rt superior frontal gyrus & 12 & 53 & 27 & 3.54 & $<0.001$ \\
\hline Lt superior frontal gyrus & -18 & 50 & 30 & 3.43 & $<0.001$ \\
\hline Rt anterior cingular gyrus & 2 & 21 & -3 & 3.18 & 0.001 \\
\hline Rt medial frontal gyrus & 30 & 53 & 18 & 3.02 & 0.001 \\
\hline \multicolumn{6}{|c|}{ Younger adults, coupling with Rt DLPFC } \\
\hline Rt medial frontal gyrus & 9 & 51 & 15 & 7.1 & $<0.001$ \\
\hline Lt putamen & -21 & 18 & -3 & 5.75 & $<0.001$ \\
\hline Lt inferior frontal gyrus & -27 & 27 & -9 & 5.48 & $<0.001$ \\
\hline Rt inferior frontal gyrus & 51 & 42 & 9 & 3.79 & 0.001 \\
\hline Rt caudate & 12 & 12 & 3 & 3.76 & 0.001 \\
\hline Lt caudate & -15 & -3 & 24 & 3.11 & 0.004 \\
\hline Rt paracentral lobule & 6 & -36 & 75 & 3.71 & 0.001 \\
\hline Rt precentral gyrus & 63 & 9 & 21 & 3.49 & 0.002 \\
\hline Lt postcentral gyrus & -42 & -30 & 66 & 3.28 & 0.003 \\
\hline Rt postcentral gyrus & 39 & -36 & 63 & 3.16 & 0.003 \\
\hline Lt medial temporal lobule & -60 & -9 & -12 & 3.12 & 0.004 \\
\hline Lt anterior cingular gyrus & -42 & 36 & 21 & 3.08 & 0.004 \\
\hline
\end{tabular}

Coordinates are in MNI space (Evans et al. 1993).

Thresholded at $p<0.05$, topological false-discovery rate corrected.

Rt, right; Lt, left; Rt PMd, right dorsal premotor cortex; Rt DLPFC, right dorsal lateral prefrontal cortex.

RT during the retention test following IP. Figure 5 demonstrated that the PPI effects of the interleaved versus the repetitive condition on functional connectivity significantly correlated with enhanced learning on day 5 , and the pattern of this connectivity-behavioral association was qualitatively different between younger and older adults. In younger adults, we identified a positive correlation between the learning benefit of IP and the increased regression slopes of BOLD activity for DLPFC with SMA, and DLPFC with the IFL $[r=0.691, p=0.003$, Fig. 5I(D); $r=0.624, p=0.005$, Fig. 5I(E) $]$. This finding suggests that increased DLPFC connectivity with the SMA and the inferior frontal region supports more efficient retrieval of motor memory following IP in younger adults. However, CI benefits on retention in older adults were associated with other brain circuits, such as those connecting PMd and IPL, DLPFC and the rostral frontal lobule (RFL), and the right and left DLPFC. In these three pairs of regions, greater interleaved-minus-repetitive differences in regression slopes of BOLD activity were associated with better retention following IP [PMd-IPL: $r=0.564, p=0.011$, Fig. 5II(A); DLPFC-RFL: $r=0.506, p=0.023$; Fig. 5II(B); Right DLPFC-Left DLPFC: $r=0.438, p=0.045$; Fig. 5II(C)].

These results overall demonstrate that contextual interference enhances cortical connectivity, which is associated with benefits to skill learning. However, the neural substrates underlying this association may differ between younger and 
FIG. 4. Age $\times$ Practice condition interaction in functional connectivity during retention. We investigated whether Age $\times$ Practice condition interactions significantly affected the connectivity with respect to the right $\mathrm{PMd}$ and the right DLFPC using the regions where significant PPI effect was found in Fig. 3 as ROIs. At each voxel of the ROIs, the PPI effects of the practice

conditions (interleaved or repetitive) with respect to the right PMd (A) or the right DLPFC (B) were compared across the age groups under a random-effects model. Colored areas in the resulting significance maps indicate brain regions with significant age-group differences, corrected for multiple comparisons. All slices are displayed in neurological orientation with the MNI coordinate at the bottom. Older adults showed greater connectivity between the right PMd and the IPL region, the right and left DLPFC, and the right DLPFC and the left IFL during sequence retrieval following IP [A(1), B(1,2)], while younger adults demonstrated greater connectivity between DLPFC and the SMA after the same context of practice [B(3)]. The Age $\times$ Practice condition interaction on functional connectivity may be more clearly demonstrated by the bar graphs that showed the differences across practice conditions ( $x$-axis) in regression slopes of BOLD activities ( $y$-axis) between the seed region and the region indicated by the arrowhead. These findings indicate an age-related difference in recruiting functional cortical networks to tackle the challenge from environmental or behavioral demands. PPI, psychophysiological interaction; IPL, inferior parietal lobule; SMA, supplementary motor area; Rt, right; Lt, left.
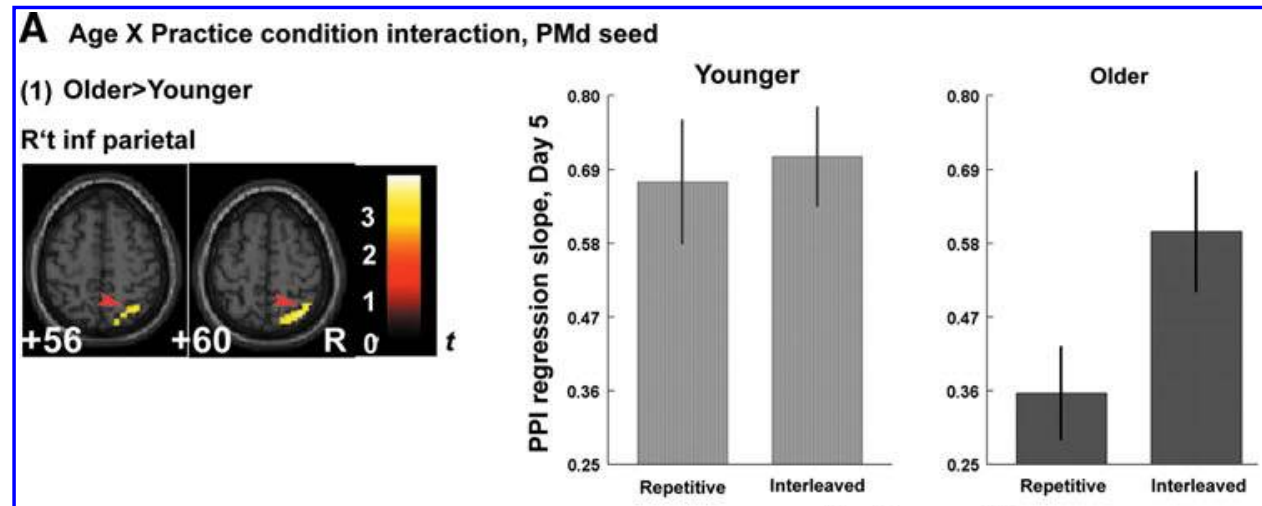

Practice condition

B Age X Practice condition interaction, Rt DLPFC seed

(1) Older>Younger
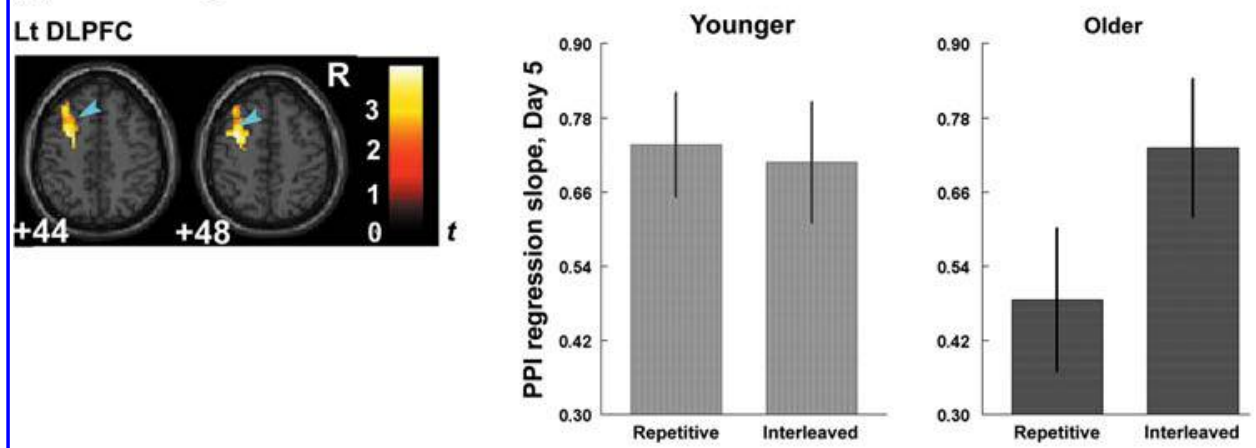

Practice condition

(2) Older>Younger

Lt IFL

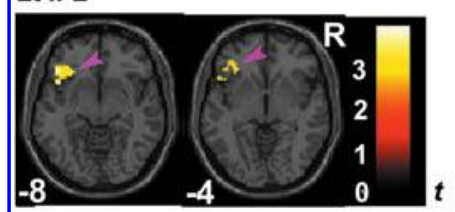

(3) Younger>Older

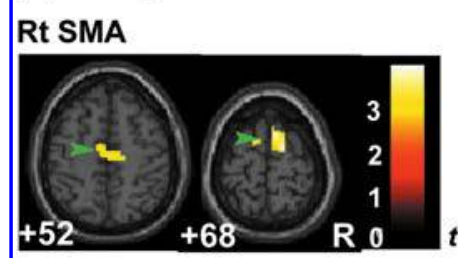

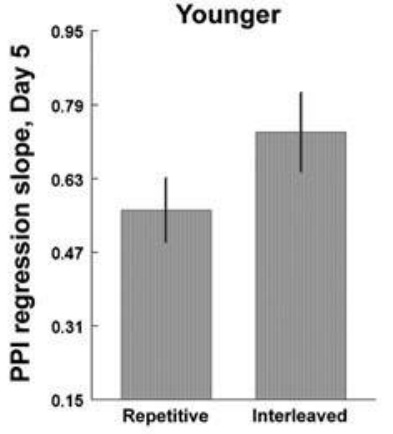

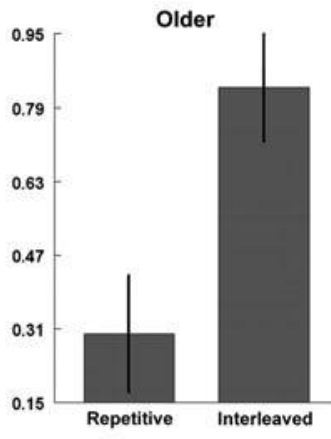

Practice condition
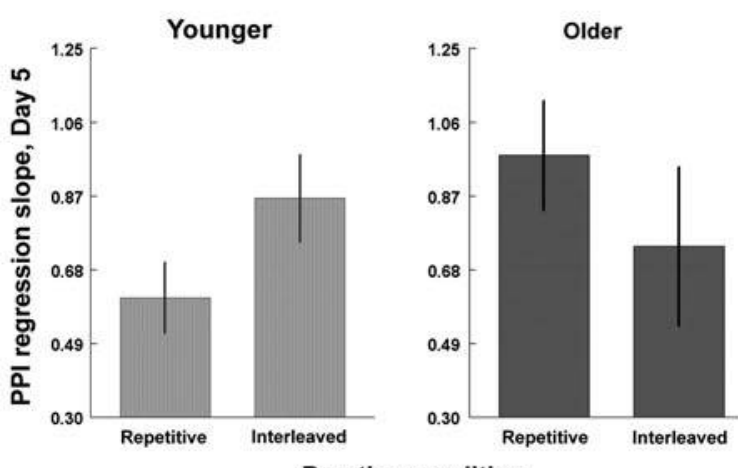

Practice condition 


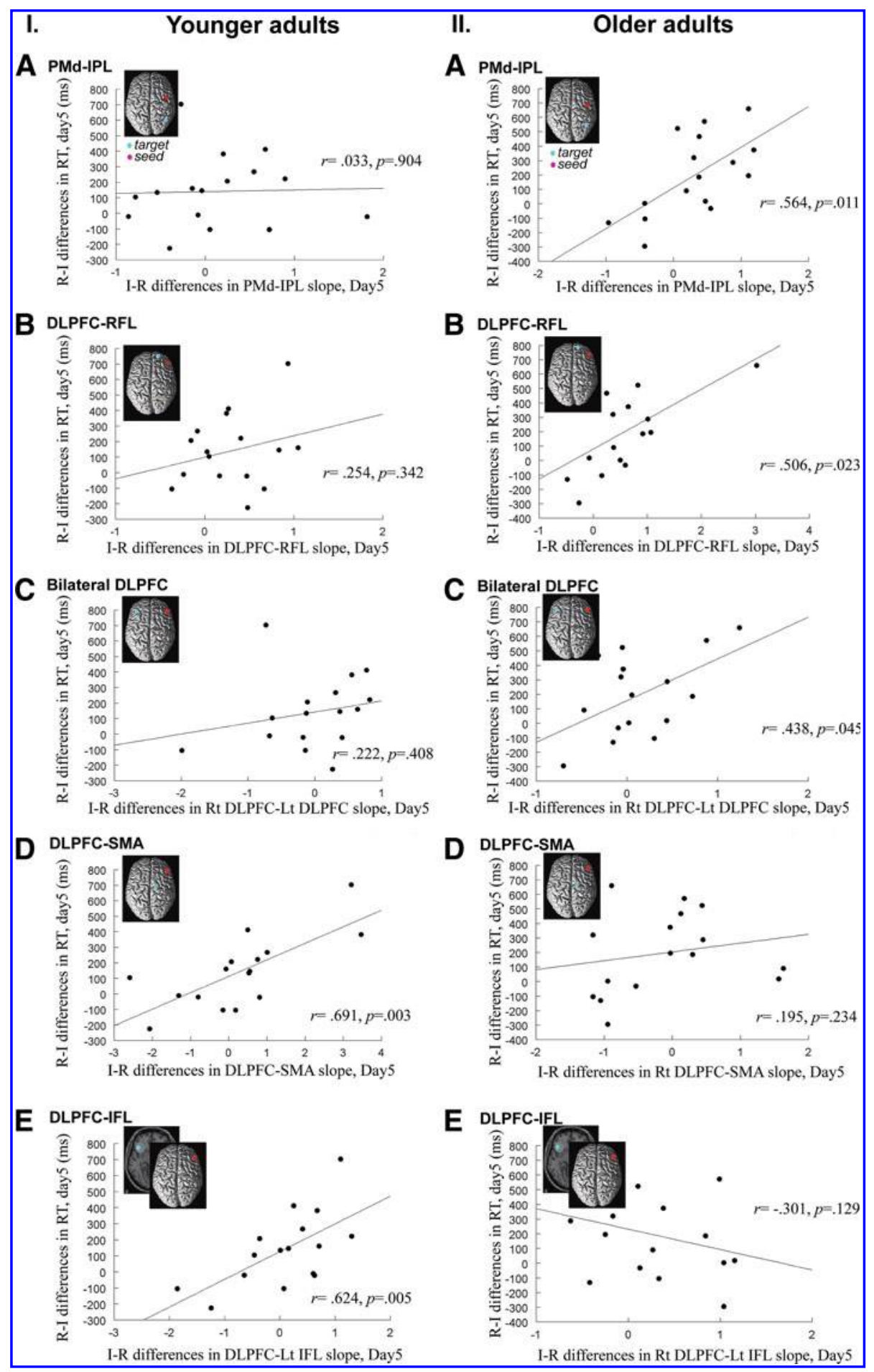

FIG. 5. Differences between older and younger adults in brain regions where increased function connectivity was associated with enhanced learning. The $x$-axis of the scatter plots indicates the difference between the IP and the RP condition in regression slopes of BOLD activities between the ROI pairs. Higher $x$-values mean greater functional connectivity between the ROIs during sequence retrieval following the IP. Learning benefit is represented by the difference in the RT between the IP and the $\mathrm{RP}$, measured on the retention testing on day 5 and displayed on the $y$-axis. Here, we used the difference in RT for the RP over the IP (note that this is opposed to the interleaved-minus-repetitive difference in the $x$-axis), as we already knew that the IP led to better learning performance in both age groups (Lin et al., 2010), and preferred to show the data within the first quadrant of the Cartesian coordinates. In the older adults, greater learning benefit of the IP over RP was associated with greater connectivity between the following ROI pairs: premotor-IPL [II(A)], DLPFC-RFL [II(B)], and bilateral DLPFC [II(C)]. The association was not significant in the DLPFC-SMA [II(D)]. For the younger adults, better learning following the IP was associated with greater connectivity between the DLPFC-SMA [I(D)] and DLPFC-IFL [I(E)]. These findings suggest that younger and older adults may rely on different brain networks for successful retrieval: younger adults need more DLPFC and SMA connectivity, while older adults require stronger connectivity within the frontal-parietal network. RFL, rostral frontal lobule; IFL, inferior frontal lobule. 
older adults. In younger adults, CI during practice appears to result in a strengthened DLPFC executive network, leading to enhanced retrieval of the encoded motor programs stored in SMA (Sun et al., 2007), and to enhanced inhibition on competing responses by the inferior frontal gyrus (Ross et al., 2010; Wendelken et al., 2009). In older adults, CI may improve retention by strengthening sensorimotor integration (PMdIPL circuitry). Moreover, enhanced interhemispheric connectivity in DLPFC is beneficial to retention in older adults, which may support the idea that recruitment of contralateral structures is a compensatory mechanism in aging (ReuterLorenz, 2002).

\section{Discussion}

In this study, we investigated whether motor learning in older adults could be optimized by IP and how the practice schedule alters functional connectivity in older and younger brains. The PMd and DLPFC were selected as a priori seed regions for the PPI analyses because of their functional significance in movement planning and executive function. By comparing the functional connectivity 3 days after sequence practice and consolidation between the two training conditions, we found that enhanced learning associated with IP was accompanied by increased DLPFC- and PMd-seeded connectivity in older adults. In contrast, in younger adults, increased connectivity as a result of IP was detected only in DLPFC, not in PMd. In addition, the increased functional connectivity following IP correlated with better retention, though younger and older adults differed in the pattern of neural correlates of these benefits. The results suggest that learning in older adults can be enhanced with appropriate arrangement of practice conditions and that this enhancement is associated with persistent changes in functional connectivity.

The behavioral data replicate the CI benefits to skill learning in both younger and older adults and confirm the feasibility of enhancing learning in the older population by introducing desirable difficulties into practice (Lin et al., 2010). Both age groups performed motor sequences more slowly during IP, but ultimately, the retention performance was superior compared to the repetitive condition. However, younger and older adults developed different brain networks to support the behavioral benefits of interleaved training. When performing the trained sequences 3 days after IP, connectivity of both DLPFC and PMd networks was strengthened in older adults, whereas in younger adults, only greater DLPFC connectivity, not PMd connectivity, was present (Fig. 3). The pattern of correlations between enhanced learning associated with IP and increased functional connectivity also differed between younger and older adults (Fig. 5). For the younger adults, better retention was associated with increased connectivity between the right DLPFC and the SMA, and between the right DLPFC and the IFL, but not with the PMd networks. The correlation between increased connectivity and better learning suggests that the benefits of $\mathrm{CI}$ in younger group may be mediated by the DLPFC executive network, where enhanced retention could be achieved by efficiently retrieving the encoded motor programs that were stored in SMA (Sun et al., 2007), and by suppressing competing responses by engaging the inferior frontal gyrus (Wendelken et al., 2009).
By contrast, in older adults, better retention was associated with both DLPFC and PMd networks, including increased connectivity between the right and left DLPFC, and between the right PMd and the IPL. The bilateral prefrontal involvement in older adults and its functional significance in learning are consistent with the aging-imaging literature where the prefrontal lateralization of older adults appears to be reduced and bilateral activation patterns occur during retrieval (Cabeza et al., 2004; Gutchess et al., 2005). It also supports previous investigations, where older adults showed stronger activation than the young adults in the left prefrontal cortex during recognition or recall tests. More importantly, our results suggest that changes in DLPFC connectivity play a unique role in good retention regardless of age, though DLPFC of younger and older adults may interact with different brain regions to support such learning benefits.

Unlike younger adults, increased PMd connectivity was present in older adults after interleaved training. This increased connectivity between the right PMd and the IPL was associated with better retention performance [Fig. 5, II(A)]. The results support previous studies showing that greater premotor, parietal, and DLPFC activity is present in older adults compared to young subjects when performing delayed retrieval (Zarahn et al., 2007) or motor tasks (Wu and Hallett, 2005). Instead of retrieving learned motor sequences using the DLPFC-SMA network like younger adults, older adults may rely on sensorimotor processing during retrieval as if they still planned and prepared to execute the task sequences that they had practiced 3 days ago. This may also indicate that older adults did not develop or consolidate their motor representations as well as their younger peers. Consequently, increased sensorimotor processing (by parietal region) and planning (by premotor area) may help older adults achieve the CI benefits on retention.

Previous studies have demonstrated that inter-regional brain connectivity can change rapidly after just one session of motor practice (Wu et al., 2008), and then evolve gradually over the next few days (Song et al., 2011), and may last for several weeks (Ma et al., 2010). Functional connectivity has been shown to predict how well an individual learns cognitive or motor tasks (Henson et al., 1999; Wu et al., 2008) and may index functional decline as a result of aging (Cook et al., 2007). For instance, older adults exhibited greater prefrontal connectivity in semantic tasks (Cook et al., 2007) and altered hippocampal-prefrontal connectivity in memory performance compared to younger adults (Grady et al., 2003). These studies validate the use of connectivity analyses to identify age-related neural changes during skill learning and retrieval.

\section{Conclusion}

We demonstrated that enhanced learning in older adults is associated with a persistent increase in brain connectivity, including increased bilateral prefrontal involvement, which may indicate decreased hemispheric laterality in older adults, and increased premotor-parietal connectivity, which may signal functional compensation. Our interpretation is, however, limited by the small sample size and limited number of $a$ priori seed regions, which makes it difficult to generalize our findings to other cerebral areas. Future studies may implement 
neural modulation techniques, or examine patients with neurological disorders, to validate the role of dorsal premotor and DLPFC and their distinct contributions to the CI effect.

\section{Author Disclosure Statement}

No competing financial interests exist.

\section{References}

Aron AR, Robbins TW, et al. 2004. Inhibition and the right inferior frontal cortex. Trends Cogn Sci 8:170-177.

Brady F. 2008. The contextual interference effect and sport skills. Percept Motor Skill 106:461-472.

Cabeza R, Daselaar SM, et al. 2004. Task-independent and taskspecific age effects on brain activity during working memory, visual attention and episodic retrieval. Cereb Cortex 14:364375.

Cabeza R, McIntosh AR, et al. 1997. Age-related differences in effective neural connectivity during encoding and recall. Neuroreport 8:3479-3483.

Chumbley J, Worsley K, et al. 2010. Topological FDR for neuroimaging. Neuroimage 49:3057-3064.

Cook IA, Bookheimer SY, et al. 2007. Aging and brain activation with working memory tasks: an fMRI study of connectivity. Int J Geriatr Psychiatry 22:332-342.

Cross ES, Schmitt PJ, et al. 2007. Neural substrates of contextual interference during motor learning support a model of active preparation. J Cogn Neurosci 19:1854-1871.

Evans AC, Collins DL, et al. 1993. 3D Statistical Neuroanatomical Models from 305 MRI Volumes. Nuclear Science Symposium and Medical Imaging Conference, 1993, 1993 IEEE Conference Record, Piscataway, NJ.

Folstein MF, Folstein SE, et al. 1975. Mini-mental state. A practical method for grading the cognitive state of patients for the clinician. J Psychiatr Res 12:189-198.

Friston KJ. 1994. Functional and effective connectivity in neuroimaging: a synthesis. Hum Brain Mapp 2:56.

Friston KJ, Buechel C, et al. 1997. Psychophysiological and modulatory interactions in neuroimaging. Neuroimage 6:218-229.

Friston KJ, Holmes AP, et al. 1995. Analysis of fMRI time-series revisited. Neuroimage 2:45-53.

Friston KJ, Stephan KE, et al. 2005. Mixed-effects and fMRI studies. Neuroimage 24:244-252.

Gitelman DR, Penny WD, et al. 2003. Modeling regional and psychophysiologic interactions in fMRI: the importance of hemodynamic deconvolution. Neuroimage 19:200-207.

Grady CL, McIntosh AR, et al. 2003. Age-related differences in the functional connectivity of the hippocampus during memory encoding. Hippocampus 13:572-586.

Gutchess AH, Welsh RC, et al. 2005. Aging and the neural correlates of successful picture encoding: frontal activations compensate for decreased medial-temporal activity. I Cogn Neurosci 17:84-96.

Henson RN, Shallice T, et al. 1999. Right prefrontal cortex and episodic memory retrieval: a functional MRI test of the monitoring hypothesis. Brain 122 (Pt 7):1367-1381.

Karni A, Meyer G, et al. 1998. The acquisition of skilled motor performance: fast and slow experience-driven changes in primary motor cortex. Proc Natl Acad Sci U S A 95:861868.

Keisker B, Hepp-Reymond MC, et al. 2009. Differential force scaling of fine-graded power grip force in the sensorimotor network. Hum Brain Mapp 30:2453-2465.
Klostermann EC, Braskie MN, et al. 2012. Dopamine and frontostriatal networks in cognitive aging. Neurobiol Aging 33:623.e15623.e24.

Lee TD. 1988. Transfer-appropriate processing: a framework for conceptualizing practice effects in motor learning. In: Meijer OG, Roth K (eds.) Complex Movement Behaviour: The MotorAction Controversy. North-Holland: Elsevier Science Publishers B.V; pp. 201-215.

Lee TD, Magill RA. 1983. The locus of contextual interference in motor-skill acquisition. J Exp Psychol Hum Learn Mem 9:730-746.

Li K, Guo L, et al. 2009. Review of methods for functional brain connectivity detection using fMRI. Comput Med Imaging Graph 33:131-139.

Lin CH, Knowlton BJ, et al. 2011. Brain-behavior correlates of optimizing learning through interleaved practice. Neuroimage 3:1758-1772.

Lin $\mathrm{CH}, \mathrm{Wu} \mathrm{AD}$, et al. 2010. Contextual interference effects in sequence learning for young and older adults. Psychol Aging 25:929-939.

Ma L, Wang B, et al. 2010. Changes in regional activity are accompanied with changes in inter-regional connectivity during 4 weeks motor learning. Brain Res 1318:64-76.

Marek T, Fafrowicz M, et al. 2010. Diurnal patterns of activity of the orienting and executive attention neuronal networks in subjects performing a Stroop-like task: a functional magnetic resonance imaging study. Chronobiol Int 27:945-958.

Mohanty A, Gitelman DR, et al. 2008. The spatial attention network interacts with limbic and monoaminergic systems to modulate motivation-induced attention shifts. Cereb Cortex 18:2604-2613.

Reuter-Lorenz P. 2002. New visions of the aging mind and brain. Trends Cogn Sci 6:394.

Rissman J, Gazzaley A, et al. 2004. Measuring functional connectivity during distinct stages of a cognitive task. Neuroimage 23:752-763.

Ross B, Hillyard SA, et al. 2010. Temporal dynamics of selective attention during dichotic listening. Cereb Cortex 20:1360-1371.

Rubinov M, Sporns O. 2010. Complex network measures of brain connectivity: uses and interpretations. Neuroimage 52:10591069.

Rypma B, D'Esposito M. 2000. Isolating the neural mechanisms of age-related changes in human working memory. Nat Neurosci 3:509-515.

Schwarb H, Schumacher EH. 2009. Neural evidence of a role for spatial response selection in the learning of spatial sequences. Brain Res 1247:114-125.

Shea JB, Morgan R. 1979. Contextual interference effects on the acquisition, retention, and transfer of a motor skill. J Exp Psychol Hum Learn Mem 5:179-187.

Song S, Sharma N, et al. 2011. White matter microstructural correlates of superior long-term skill gained implicitly under randomized practice. Cereb Cortex. [Epub ahead of print] DOI: 10.1093 /cercor/bhr247

Sun FT, Miller LM, et al. 2007. Functional connectivity of cortical networks involved in bimanual motor sequence learning. Cereb Cortex 17:1227-1234.

Wendelken C, Ditterich J, et al. 2009. Stimulus and response conflict processing during perceptual decision making. Cogn Affect Behav Neurosci 9(4):434-447.

Wolk DA, Sen NM, et al. 2009. ERP correlates of item recognition memory: effects of age and performance. Brain Res 1250:218-231.

Wright DL, Magnuson CE, et al. 2005. Programming and reprogramming sequence timing following high and low contextual interference practice. Res Q Exerc Sports 76:258-266. 
Wu T, Chan P, et al. 2008. Modifications of the interactions in the motor networks when a movement becomes automatic. I Physiol 586(Pt 17):4295-4304.

$\mathrm{Wu}$ T, Hallett M. 2005. The influence of normal human ageing on automatic movements. J Physiol 562(Pt 2):605-615.

Zarahn E, Rakitin B, et al. 2007. Age-related changes in brain activation during a delayed item recognition task. Neurobiol Aging 28:784-798.
Address correspondence to: Barbara J. Knowlton

Department of Psychology University of California, Los Angeles 6623 Franz Hall, Los Angeles CA 90095-7332

E-mail: knowlton@psych.ucla.edu 